MATHEMATICS OF COMPUTATION

Volume 77, Number 261, January 2008, Pages 313-334

S 0025-5718(07)02005-4

Article electronically published on May 11, 2007

\title{
ON PSEUDOSPECTRA OF MATRIX POLYNOMIALS AND THEIR BOUNDARIES
}

\author{
LYONELL BOULTON, PETER LANCASTER, AND PANAYIOTIS PSARRAKOS
}

\begin{abstract}
In the first part of this paper (Sections 2-4), the main concern is with the boundary of the pseudospectrum of a matrix polynomial and, particularly, with smoothness properties of the boundary. In the second part (Sections 5-6), results are obtained concerning the number of connected components of pseudospectra, as well as results concerning matrix polynomials with multiple eigenvalues, or the proximity to such polynomials.
\end{abstract}

\section{INTRODUCTION}

This paper falls into two parts. In the first (Sections 244), the main concern is with the boundary of the pseudospectrum of a matrix polynomial and, particularly, in view of its importance for boundary-tracing algorithms, with the smoothness properties of the boundary. In the second (Sections 5 58), we further develop analysis begun by two of the present authors (see [12]) on qualitative aspects of the pseudospectrum. This part is also influenced by earlier work on pseudospectra for standard eigenvalue problems by Alam and Bora in 2. In particular, results are presented concerning the number of connected components of the pseudospectrum and proximity to systems with multiple eigenvalues.

Let us begin with some formal definitions. First, a matrix polynomial is a function $P: \mathbb{C} \rightarrow \mathbb{C}^{n \times n}$ (the algebra of all $n \times n$ complex matrices) of the form

$$
P(\lambda)=P_{m} \lambda^{m}+P_{m-1} \lambda^{m-1}+\cdots+P_{1} \lambda+P_{0},
$$

where $\lambda$ is a complex variable and $P_{0}, P_{1}, \ldots, P_{m} \in \mathbb{C}^{n \times n}$ with $\operatorname{det} P_{m} \neq 0$. The spectrum of such a function is $\sigma(P):=\{\lambda \in \mathbb{C}: \operatorname{det} P(\lambda)=0\}$.

Since $\operatorname{det} P_{m} \neq 0, \sigma(P)$ consists of no more than $n m$ distinct eigenvalues. A nonzero vector $x_{0} \in \mathbb{C}^{n}$ is known as an eigenvector of $P(\lambda)$ corresponding to an eigenvalue $\lambda_{0} \in \sigma(P)$ if it satisfies $P\left(\lambda_{0}\right) x_{0}=0$. The algebraic multiplicity of a $\lambda_{0} \in \sigma(P)$ is the multiplicity of $\lambda_{0}$ as a zero of the scalar polynomial $\operatorname{det} P(\lambda)$, and it is always greater than or equal to the geometric multiplicity of $\lambda_{0}$, that is, the dimension of the null space of the matrix $P\left(\lambda_{0}\right)$. A multiple eigenvalue of $P(\lambda)$ is called defective if its algebraic multiplicity exceeds its geometric multiplicity.

We let $\mathcal{P}_{m}$ denote the linear space of $n \times n$ matrix polynomials with degree $m$ or less. Using the spectral matrix norm (i.e., that norm subordinate to the Euclidean

Received by the editor April 6, 2006 and, in revised form, October 29, 2006.

2000 Mathematics Subject Classification. Primary 65F15; Secondary 65F35, 93D09.

Key words and phrases. Matrix polynomials, perturbation of eigenvalues, singular values, pseudospectra. 
vector norm), we may define the max norm on $\mathcal{P}_{m}$,

$$
\|P(\lambda)\|:=\max _{0 \leq j \leq m}\left\|P_{j}\right\| .
$$

Using this norm, we construct a class of matrix polynomials obtained from $P(\lambda)$ in (11) by perturbation. The admissible perturbations are defined in terms of a real polynomial $w(x)=\sum_{j=0}^{m} w_{j} x^{j}$ with nonnegative coefficients and a positive constant coefficient; $w_{j} \geq 0$ for each $j=1,2, \ldots, m$, and $w_{0}>0$. First consider matrix polynomials in $\mathcal{P}_{m}$ of the form

$$
Q(\lambda)=\left(P_{m}+\Delta_{m}\right) \lambda^{m}+\cdots+\left(P_{1}+\Delta_{1}\right) \lambda+\left(P_{0}+\Delta_{0}\right)
$$

where the matrices $\Delta_{j} \in \mathbb{C}^{n \times n}(j=0,1, \ldots, m)$ are arbitrary. Then, for a given $\varepsilon \geq 0$, the class of admissible perturbed matrix polynomials is

$$
\mathcal{B}(P, \varepsilon, w):=\left\{Q(\lambda):\left\|\Delta_{j}\right\| \leq \varepsilon w_{j}, j=0,1, \ldots, m\right\} .
$$

This is a convex compact set in the linear space $\mathcal{P}_{m}$ with the norm (2).

The $\varepsilon$-pseudospectrum of $P(\lambda)$ with respect to $w(x)$ (introduced by Tisseur and Higham [17]) is then

$$
\Lambda_{\varepsilon}(P):=\{\mu \in \mathbb{C}: \operatorname{det} Q(\mu)=0 \text { for some } Q(\lambda) \in \mathcal{B}(P, \varepsilon, w)\} .
$$

As $w(x)$ is generally fixed throughout this paper, it will not appear explicitly in this notation, and we will refer to $\Lambda_{\varepsilon}(P)$ simply as the $\varepsilon$-pseudospectrum of $P(\lambda)$. Note that if $\varepsilon w_{m}<\left\|P_{m}^{-1}\right\|^{-1}$, then all matrix polynomials in $\mathcal{B}(P, \varepsilon, w)$ have nonsingular leading coefficients, and this ensures that $\Lambda_{\varepsilon}(P)$ is bounded (Theorem 2.2 of [12]).

If we define the standard eigenvalue problem as that in which $P(\lambda)=I \lambda-A$, then it is natural to define weights $w_{1}=0$ (no perturbation of the coefficient $I$ is admitted) and $w_{0}=1$. Thus, $w(x)=1$ and, using (5), we obtain the relatively well-understood " $\varepsilon$-pseudospectrum of matrix $A$ ", namely,

$$
\Lambda_{\varepsilon}(A) \equiv \Lambda_{\varepsilon}(P)=\left\{\mu \in \mathbb{C}: \operatorname{det}\left(I \mu-\left(A+\Delta_{0}\right)\right)=0,\left\|\Delta_{0}\right\| \leq \varepsilon\right\} .
$$

\section{The Singular Value functions}

For any $\lambda \in \mathbb{C}$, the singular values of a matrix polynomial $P(\lambda)$ are the nonnegative square-roots of the $n$ eigenvalue functions of $P(\lambda)^{*} P(\lambda)$. They are denoted by

$$
s_{1}(\lambda) \geq s_{2}(\lambda) \geq \cdots \geq s_{n}(\lambda) \geq 0 .
$$

The real-valued function $s_{n}: \mathbb{C} \longrightarrow[0, \infty)$, given by the smallest singular value, provides more information about the matrix polynomial $P(\lambda)$ than $\sigma(P)$ alone. This will become clear in the forthcoming section when we discuss the pseudospectrum of $P(\lambda)$. Let us first describe some general properties of $s_{n}(\lambda)$.

It is clear that an alternate definition of the spectrum of a matrix polynomial $P(\lambda)$ is:

$$
\sigma(P)=\left\{\lambda \in \mathbb{C}: s_{n}(\lambda)=0\right\} .
$$

The connection between the zeros of $s_{n}(\lambda)$ and the eigenvalues of $P(\lambda)$ can be made more precise using the singular value decomposition.

Proposition 1. An eigenvalue $\lambda_{0} \in \sigma(P)$ has geometric multiplicity $k$ if and only if

$$
s_{1}\left(\lambda_{0}\right) \geq s_{2}\left(\lambda_{0}\right) \geq \cdots \geq s_{n-k}\left(\lambda_{0}\right)>s_{n-k+1}\left(\lambda_{0}\right)=\cdots=s_{n}\left(\lambda_{0}\right)=0 .
$$


Our analysis depends on an important, concise characterisation of the $\varepsilon$-pseudospectrum in terms of the function $s_{n}(\lambda)$. This was obtained by Tisseur and Higham (Lemma 2.1 of [17]),

$$
\Lambda_{\varepsilon}(P)=\left\{\lambda \in \mathbb{C}: s_{n}(\lambda) \leq \varepsilon w(|\lambda|)\right\} .
$$

Clearly, $\sigma(P)=\Lambda_{0}(P) \subset \Lambda_{\varepsilon}(P)$ for any $\varepsilon>0$. Thus, $\Lambda_{\varepsilon}(P)$ is nothing but the level set at height 0 of the real-valued function $s_{n}(\lambda)-\varepsilon w(|\lambda|)$, or that at height $\varepsilon$ of the function $s_{n}(\lambda) w(|\lambda|)^{-1}$. Notice also that in the standard eigenvalue problem, $\varepsilon w(|\lambda|)=\varepsilon$ in (6). More generally, $\varepsilon w(|\lambda|)$ (in equation ([6) ) is a radially symmetric nondecreasing function of $\lambda$.

By using the Euclidean vector norm,

$$
s_{n}(\lambda)=\min _{u \neq 0} \frac{\|P(\lambda) u\|}{\|u\|} .
$$

Our first theorem has been originally established by Davies in the more general context of holomorphic families of bounded operators. A proof is included here for completeness.

Theorem 2. Let $P(\lambda)$ be invertible on a domain $U$. Then $s_{n}(\lambda)^{-1}$ is a subharmonic function on $U$.

Proof. First we recall one of the characterisations of continuous subharmonic functions (see Ahlfors [1, for example). A continuous function $\phi: U \rightarrow \mathbb{R}$ is subharmonic if and only if, for any closed disc in $U$ with centre $\lambda_{0}$ and radius $r$,

$$
\phi\left(\lambda_{0}\right) \leq \frac{1}{2 \pi} \int_{0}^{2 \pi} \phi\left(\lambda_{0}+r e^{i \theta}\right) d \theta
$$

A well known result from operator theory establishes that for any bounded linear operator $T$ on a Hilbert space,

$$
\|T\|=\sup _{\phi, \psi \neq 0} \frac{\operatorname{Re}\langle T \phi, \psi\rangle}{\|\phi\|\|\psi\|} .
$$

If the Hilbert space is finite dimensional, it is easy to see that the supremum is attained. By virtue of (7), $s_{n}(\lambda)=\left\|P(\lambda)^{-1}\right\|^{-1}$. Thus,

$$
s_{n}(\lambda)=\left[\max _{u, v \neq 0} \frac{\operatorname{Re}\left\langle P(\lambda)^{-1} u, v\right\rangle}{\|u\|\|v\|}\right]^{-1} .
$$

Now note that $s_{n}(\lambda)^{-1}$ is continuous on $U$, and let $\lambda_{0} \in U$ and $u_{0}, v_{0} \in \mathbb{C}^{n}$ be such that

$$
s_{n}\left(\lambda_{0}\right)^{-1}=\frac{\operatorname{Re}\left\langle P\left(\lambda_{0}\right)^{-1} u_{0}, v_{0}\right\rangle}{\left\|u_{0}\right\|\left\|v_{0}\right\|} .
$$

The function $\left\langle P(\lambda)^{-1} u_{0}, v_{0}\right\rangle$ is analytic on $U$ and so the real function

$$
h(\lambda):=\frac{\operatorname{Re}\left\langle P(\lambda)^{-1} u_{0}, v_{0}\right\rangle}{\left\|u_{0}\right\|\left\|v_{0}\right\|}
$$

is harmonic on $U$. Furthermore, it follows from (8) and (9) that $h(\lambda) \leq s_{n}(\lambda)^{-1}$ on $U$. Consequently,

$$
s_{n}\left(\lambda_{0}\right)^{-1}=h\left(\lambda_{0}\right)=\frac{1}{2 \pi} \int_{0}^{2 \pi} h\left(\lambda_{0}+r e^{i \theta}\right) d \theta \leq \frac{1}{2 \pi} \int_{0}^{2 \pi} s_{n}\left(\lambda_{0}+r e^{i \theta}\right)^{-1} d \theta,
$$

and the result follows. 
An important characteristic of subharmonic functions is the fact that they satisfy the maximum principle. Therefore, the only local minima of $s_{n}(\lambda)$ are those $\lambda \in$ $\sigma(P)$.

The subharmonicity of $s_{n}(\lambda)^{-1}$ has been considered recently by various authors. In [4, Boyd and Desoer discuss this property in the context of linear control systems. Concrete applications of this theorem may be found in [8] for the linear case, and in [3, 6] for the quadratic case. In [3, the result is applied in support of a certain novel procedure for finding eigenvalues of self-adjoint operators in infinite dimensional Hilbert spaces.

Corollary 3. For all $\varepsilon>0$, every connected component of $\Lambda_{\varepsilon}(P)$ has nonempty interior.

Proof. Suppose, on the contrary, that $\mathcal{G}$ is a connected component of $\Lambda_{\varepsilon}(P)$ with empty interior. Since $w(|\lambda|) / s_{n}(\lambda)$ is subharmonic, $\min _{\lambda \in \mathcal{G}}\left[s_{n}(\lambda) / w(|\lambda|)\right]$ should be attained at all points of $\mathcal{G}$. Thus, necessarily, $\mathcal{G}$ should be a single point and in fact one of the eigenvalues of $P(\lambda)$. The continuity of $s_{n}(\lambda)$ and the fact that $w(0)=w_{0}>0$ ensure that this is not possible.

In general, $s_{n}(\lambda)$ itself is not a subharmonic function, as it does not satisfy the maximum principle (a concrete example may be found at the end of this section). However, as we will see next, $s_{n}(x)$ is locally regular.

First consider the nonnegative eigenvalue functions generated on $\mathbb{C}$ by the matrix function $P(\lambda)^{*} P(\lambda)$, say $S_{1}(\lambda), S_{2}(\lambda), \ldots, S_{n}(\lambda)$. They can be organised in such a way that they have a strong smoothness property.

Lemma 4. For any given analytic curve $\zeta: \mathbb{R} \rightarrow \mathbb{C}$, the eigenvalues of $P(\lambda)^{*} P(\lambda)$ can be arranged in such way that, for all $j, S_{j}(\zeta(t))$ are real analytic functions of $t \in \mathbb{R}$.

Furthermore, if $s_{n}(\lambda)=\min _{j}\left(S_{j}(\lambda)\right)^{1 / 2}$ is a nonzero simple singular value of $P(\lambda)$ and $u_{\lambda}, v_{\lambda}$ are associated left and right singular vectors, respectively, then (writing $\lambda=x+i y) s_{n}(\cdot)$ is a real analytic function in a neighbourhood of $\lambda$ and

$$
\nabla s_{n}(x+i y)=\left(\operatorname{Re}\left(u_{\lambda}^{*} \frac{\partial P(x+i y)}{\partial x} v_{\lambda}\right), \operatorname{Re}\left(u_{\lambda}^{*} \frac{\partial P(x+i y)}{\partial y} v_{\lambda}\right)\right) .
$$

The first statement follows from Theorem S6.3 of [9] (see also Theorem II-6.1 of [10]). For the second and third, see [15].

We can interpret the first part of this lemma pictorially in the following manner. For $t \in \mathbb{R}$, the graphs of $S_{j}(\zeta(t))(j=1,2, \ldots, n)$ are smooth and might cross each other. At a crossing point, the graph of the corresponding singular value $s_{k}(\zeta(t))$ is continuous but it changes from one smooth curve to another with a possible jump in the derivative (see $\S$ II-6.4 of [10].)

We may also consider regularity properties of $s_{n}(\lambda)$ as a function defined on the complex plane. In this case, some rudimentary ideas from algebraic geometry assist in discussing the $n$ surfaces in $\mathbb{R}^{3}$ which are (in general) generated by the singular values. (Where possible, the terminology of Kendig [11] is followed). Write $\lambda \in \mathbb{C}$ in real and imaginary parts; $\lambda=x+i y$, and define $n$ subsets of $\mathbb{R}^{3}$ :

$$
\Sigma_{j}:=\left(x, y, S_{j}(x+i y)\right), j=1,2, \ldots, n .
$$

Proposition 5. The union $\bigcup_{j=1}^{n} \Sigma_{j}$ is a real algebraic variety. 
Proof. Define the function

$$
d(x, y, S):=\operatorname{det}\left(I S-P(x+i y)^{*} P(x+i y)\right), \quad x, y, S \in \mathbb{R} .
$$

Since the matrix $P(x+i y)^{*} P(x+i y)$ is hermitian, $d(x, y, S)$ is a polynomial in $x, y, S$ with real coefficients, and since

$$
\bigcup_{j=1}^{n} \Sigma_{j}=\left\{(x, y, S) \in \mathbb{R}^{3}: d(x, y, S)=0\right\}
$$

the result follows.

In spite of this proposition and Lemma 4 , the existence of an arrangement of the eigenvalues of $P(\lambda)^{*} P(\lambda)$ such that the $n$ surfaces $\Sigma_{j} \in \mathbb{R}^{3}$ are smooth everywhere is not guaranteed in general. Consider the following example. For the linear matrix polynomial $P(\lambda)=I \lambda-A$, where

$$
A=\left[\begin{array}{ccc}
3 / 4 & 1 & 1 \\
0 & 5 / 4 & 1 \\
0 & 0 & -3 / 4
\end{array}\right],
$$

$\Sigma_{1} \cup \Sigma_{2} \cup \Sigma_{3}$ has a conic double point at $(0,0,5 / 16)$. Therefore, no arrangement of the singular values exists ensuring $\Sigma_{1}, \Sigma_{2}$ and $\Sigma_{3}$ are simultaneously smooth at $\lambda=0$. Moreover, in this example, $s_{3}(0)=s_{2}(0)=\sqrt{5 / 16}$, so note that the hypothesis of nondegeneracy of the fundamental singular value in the second part of Lemma 4 is essential.

For linear polynomials, the occurrence of isolated singularities in $\bigcup_{j=1}^{n} \Sigma_{j}$ is rare. In the above example the matrix $A$ had to be carefully crafted to allow the conic double point around the origin. Any slight change in the coefficients of $A$ would eliminate this degeneracy.

The following useful proposition is an immediate consequence of Lemma 4 .

Proposition 6. If $S_{j}(\lambda)=S_{k}(\lambda)$ for $j \neq k$ and for all $\lambda$ in a nonempty open set $\mathcal{O}$, then $\mathcal{O}=\mathbb{C}$.

Thus, different surfaces $\Sigma_{j}$ can intersect only in sets of topological dimension at most one.

\section{The PSEUdospectrum AND ITS BOUndaRY}

Now we put these ideas into the context of the study of pseudospectra.

A fundamentally important case is that in which $\varepsilon>0$ is so small that $\Lambda_{\varepsilon}(P)$ consists of "small" disconnected components, each one containing a single (possibly multiple) eigenvalue of $P(\lambda)$. As $\varepsilon$ is increased from zero, these components enlarge, collide and eventually intersect in various ways so that the boundary of $\Lambda_{\varepsilon}(P)$, say $\partial \Lambda_{\varepsilon}(P)$, becomes more complex. In an earlier paper 12 two of the present authors studied some basic properties of $\Lambda_{\varepsilon}(P)$ and $\partial \Lambda_{\varepsilon}(P)$ in support of a curve-tracing algorithm for plotting $\partial \Lambda_{\varepsilon}(P)$.

Let

$$
F_{\varepsilon}(x, y) \equiv F_{\varepsilon}(x+i y):=s_{n}(x+i y)-\varepsilon w(|x+i y|), \quad x, y \in \mathbb{R} .
$$

Since this function is continuous in $\lambda=x+i y \in \mathbb{C}$, it follows from (66) that

$$
\partial \Lambda_{\varepsilon}(P) \subseteq\left\{\lambda \in \mathbb{C}: F_{\varepsilon}(\lambda)=0\right\} .
$$


Moreover, as long as $s_{n}(\lambda)^{2}$ is a simple nonvanishing eigenvalue of $P(\lambda)^{*} P(\lambda)$, differentiation in the direction of the boundary will be well-defined as a consequence of Lemma 4. However, when $s_{n-1}(\lambda)=s_{n}(\lambda)$, this smoothness of the boundary may be lost. Hence our interest in the set of $\lambda \in \mathbb{C}$ for which $s_{n}(\lambda)$ is multiple; curve tracing algorithms are prone to fail around these points, as the directional derivatives along $\partial \Lambda_{\varepsilon}(P)$ may not be well-defined.

Even though it is quite rare 1 in general, the right side of (13) might include points in the interior of $\Lambda_{\varepsilon}(P)$. This can be observed as a consequence of either of the two unlikely events:

(i) the surface $s_{n}(\lambda)$ having a local (but not global) maximum,

(ii) at least three multiple sheets of $\bigcup_{j=1}^{n} \Sigma_{j}$ intersecting in a single point.

Demmel's matrix

$$
A=\left(\begin{array}{ccc}
-1 & -b & -b^{2} \\
0 & -1 & -b \\
0 & 0 & -1
\end{array}\right)
$$

with $b \gg 1$ illustrates (i) for the standard eigenvalue problem with $w(x)=1$. Indeed if $b=100$ and $P(\lambda)=(\lambda I-A), s_{3}(\lambda)$ has a local maximum at $\lambda=0$; cf. [7.

Higher order examples typifying (i) can also be easily constructed. Consider, for instance, the polynomial $P(\lambda)=\left(\lambda^{2}-1\right)\left(\lambda^{2}-i\right)$ in $\mathbb{C}$ and the weight function $w(x)=4 x^{2}+1$. The point $\lambda=0$ is a local maximum of the function

$$
\frac{s_{1}(\lambda)}{w(|\lambda|)}=\frac{\left|\lambda^{2}-1\right|\left|\lambda^{2}-i\right|}{4|\lambda|^{2}+1},
$$

which is smooth in $\mathbb{C} \backslash\left\{ \pm 1, \pm i^{1 / 2}\right\}$. This may be verified by directly computing the gradient and Hessian of this expression at $\lambda=0$. Thus, when $\varepsilon=1$ and $\lambda$ lies in a sufficiently small neighbourhood $\mathcal{N}$ of the origin we have $s_{1}(\lambda) \leq w(|\lambda|)$, so that $\mathcal{N} \in \Lambda_{1}(P)$ and $0 \notin \partial \Lambda_{1}(P)$. However, for $\varepsilon=1$,

$$
F_{\varepsilon}(0)=F_{1}(0)=s_{1}(0)-w(0)=0,
$$

so in this case the inclusion of (13) is proper.

To confirm (ii), recall Example 3.5 of 2 : for $w(x)=1$ and any $\varepsilon>0$, the $\varepsilon$-pseudospectrum of $P(\lambda)=\operatorname{diag}\{\lambda-1, \lambda+1, \lambda-i, \lambda+i\}$ is the union of four closed discs with centres at the eigenvalues $1,-1, i,-i$ and radii equal to $\varepsilon$. Thus, for $\varepsilon=1$, the origin lies in the set $\left\{\lambda \in \mathbb{C}: F_{1}(\lambda)=0\right\}$, but it is an interior point of $\Lambda_{1}(P)$.

The next result shows that $\partial \Lambda_{\varepsilon}(P)$ is made up of algebraic curves. This is a comforting property in the sense that the number of difficult points, such as cusps or self-intersections, is limited. (See Proposition 6.2.10 of [5] for an explicit statement of this kind.)

Theorem 7. Let $\varepsilon>0$ and assume that $\Lambda_{\varepsilon}(P) \neq \mathbb{C}$. Then the boundary of $\Lambda_{\varepsilon}(P)$ lies on an algebraic curve. In particular, $\partial \Lambda_{\varepsilon}(P)$ is a piecewise $C^{\infty}$ curve, it has at most a finite number of singularities where the tangent fails to exist, and it intersects itself only at a finite number of points.

\footnotetext{
${ }^{1}$ This is a rather delicate point, and it seems to have been missed in the work of several preceding authors as in [12] and [17. In particular, Corollary 4.3 of 2 seems to be false as it stands. On the other hand, this fact seems to have little, if any impact on the design of algorithms.
} 
Proof. We first show that $\partial \Lambda_{\varepsilon}(P)$ lies on an algebraic curve. Recall the function $d(x, y, S)$ defined by (11) and observe that $\partial \Lambda_{\varepsilon}(P)$ lies on the level set

$$
\begin{aligned}
\mathcal{L}_{1} & =\{x+i y: x, y \in \mathbb{R}, \varepsilon w(|x+i y|) \text { is a singular value of } P(x+i y)\} \\
& =\left\{x+i y: x, y \in \mathbb{R}, d\left(x, y, \varepsilon^{2} w(|x+i y|)^{2}\right)=0\right\} .
\end{aligned}
$$

The function $d\left(x, y, \varepsilon^{2} w(|x+i y|)^{2}\right)$ can be written in the form

$$
d\left(x, y, \varepsilon^{2} w(|x+i y|)^{2}\right)=\sqrt{x^{2}+y^{2}} p(x, y)+q(x, y),
$$

where $p(x, y)$ and $q(x, y)$ are real polynomials in $x, y \in \mathbb{R}$. Thus,

$$
\mathcal{L}_{1}=\left\{x+i y: x, y \in \mathbb{R}, \sqrt{x^{2}+y^{2}} p(x, y)+q(x, y)=0\right\} .
$$

If $w(x)$ is an even function, then $p(x, y)$ is identically zero and either $\mathcal{L}_{1}$ is an algebraic curve or it coincides with the complex plane. Suppose $w(x)$ is not an even function. Then $\mathcal{L}_{1}$ is a subset of the level set

$$
\mathcal{L}_{2}:=\left\{x+i y: x, y \in \mathbb{R},\left(x^{2}+y^{2}\right) p(x, y)^{2}-q(x, y)^{2}=0\right\},
$$

which is also an algebraic curve when it does not coincide with the complex plane.

Next we show that $\mathcal{L}_{2}=\mathbb{C}$ only if $\mathcal{L}_{1}=\mathbb{C}$. Thus, if $\mathcal{L}_{2}=\mathbb{C}$ and $p(x, y), q(x, y)$ are not identically zero, then

$$
\left(x^{2}+y^{2}\right) p(x, y)^{2}=q(x, y)^{2} \text { for all } x, y \in \mathbb{R},
$$

where the order of the (irreducible) factor $x^{2}+y^{2}$ in the left hand side is odd and the order of the same factor on the right (if any) is even. This is a contradiction. Hence, if $\mathcal{L}_{2}=\mathbb{C}$, then $p(x, y)$ and $q(x, y)$ are identically zero, and consequently, $\mathcal{L}_{1}=\mathbb{C}$.

Since $\mathcal{L}_{1} \subseteq \Lambda_{\varepsilon}(P)$ and by hypothesis $\Lambda_{\varepsilon}(P) \neq \mathbb{C}$, both $\mathcal{L}_{1}, \mathcal{L}_{2} \neq \mathbb{C}$ and so $\mathcal{L}_{2}$ is an algebraic curve. This completes the first part of the theorem.

For the second part, note that, as $s_{n}(\lambda)$ is continuous in $\lambda \in \mathbb{C}, \partial \Lambda_{\varepsilon}(P)$ is a union of continuous curves. From the above considerations it follows that $\mathcal{L}_{1}$ is a piecewise $C^{\infty}$ curve and it has finitely many singularities. Then, since

$$
\partial \Lambda_{\varepsilon}(P) \subseteq \mathcal{L}_{1} \subseteq \Lambda_{\varepsilon}(P),
$$

we can actually decompose $\mathcal{L}_{1}=\bigcup_{k=1}^{h} \gamma_{k}$, where $\gamma_{k}(k=1,2, \ldots, h)$ are suitable smooth curves with the following property: $\gamma_{k} \subseteq \partial \Lambda_{\varepsilon}(P)$ for all $1 \leq k \leq j$, and $\gamma_{k} \subseteq \Lambda_{\varepsilon}(P) \backslash \partial \Lambda_{\varepsilon}(P)$ for all $j<k \leq h$. Thus, $\partial \Lambda_{\varepsilon}(P)=\bigcup_{k=1}^{j} \gamma_{k}$ as needed.

Note that for the standard eigenvalue problem, $w(x)=1$ is an even function. In this case, the above result appears in the work of Alam and Bora [2].

The following technical statements will be useful subsequently. The first one follows immediately from (13).

Lemma 8. If $0 \leq \delta<\varepsilon$, then $\partial \Lambda_{\delta}(P) \subset \Lambda_{\varepsilon}(P)$ and $\Lambda_{\delta}(P) \cap \partial \Lambda_{\varepsilon}(P)=\emptyset$.

In particular, note that $\sigma(P) \cap \partial \Lambda_{\varepsilon}(P)=\emptyset$ for any $\varepsilon>0$.

With $P(\lambda)$ as in (11), consider a perturbed matrix polynomial $Q(\lambda)$ of the form (3). It follows from the definition (4) that $Q(\lambda) \in \partial \mathcal{B}(P, \varepsilon, w)$ if and only if $\left\|\Delta_{j}\right\| \leq \varepsilon w_{j}$ for each $j$ and equality holds for at least one $j$. Now consider matrix 
polynomials in the interior of $\mathcal{B}(P, \varepsilon, w) ; \operatorname{Int}[\mathcal{B}(P, \varepsilon, w)]$. It is easily seen that $Q(\lambda) \in$ $\operatorname{Int}[\mathcal{B}(P, \varepsilon, w)]$ if and only if

$$
\begin{gathered}
\left\|\Delta_{j}\right\|<\varepsilon w_{j} \text { whenever } w_{j}>0, \text { and } \\
\Delta_{j}=0 \text { whenever } w_{j}=0 .
\end{gathered}
$$

Lemma 9. If $\mu \in \partial \Lambda_{\varepsilon}(P)$, then for any perturbation $Q(\lambda) \in \mathcal{B}(P, \varepsilon, w)$ such that $\mu \in \sigma(Q), \quad Q(\lambda) \in \partial \mathcal{B}(P, \varepsilon, w)$.

Proof. Let $\mu \in \partial \Lambda_{\varepsilon}(P)$. It suffices to show that if $\mu \in \sigma(Q)$ for a $Q(\lambda) \in \mathcal{B}(P, \varepsilon, w)$, then $\left\|\Delta_{j}\right\|=\varepsilon w_{j}$ for some $j=0,1, \ldots, m$. Indeed, if we assume the converse statement, $\left\|\Delta_{j}\right\|<\varepsilon w_{j}$ for all $j$, then $Q(\lambda) \in \mathcal{B}(P, \tilde{\varepsilon}, w)$ for some $\tilde{\varepsilon}<\varepsilon$. But since $\mu \in \sigma(Q)$, we have $\mu \in \Lambda_{\tilde{\varepsilon}}(P)$, which contradicts Lemma 8 . Thus, the desired assertion holds.

\section{The FAUlt LiNES}

Differentiability along $\partial \Lambda_{\varepsilon}(P)$, the boundary of the pseudospectrum, is possible as long as the gradient of $s_{n}(\lambda)-\varepsilon w(|\lambda|)$ exists and does not vanish. The only place where $w(|\lambda|)$ might fail to have a derivative is the origin. If the minimal singular value, $s_{n}(\lambda)$, has multiplicity one, then $s_{n}(\lambda)$ is smooth in a neighbourhood of $\lambda$. Thus, the study of those points where differentiability is lost, apart from $\lambda=0$, is confined to the region of the plane where the sheet of $\bigcup_{j=1}^{n} \Sigma_{j}$ corresponding to $s_{n}(\lambda)$ meets the one corresponding to $s_{n-1}(\lambda)$. This motivates the following definition.

The rather involved indexing introduced next is required to handle multiple eigenvalues. Below we always assume that the eigenvalues of $P(\lambda)^{*} P(\lambda)$ are ordered so that

$$
S_{j}(\lambda)=s_{j}(\lambda)^{2} \quad \text { for all } j=1,2, \ldots, n .
$$

Let $p:\{1,2, \ldots, n\} \longrightarrow\{1,2, \ldots, n\}$ (usually not onto) satisfy the following properties:

(a) $\Sigma_{j}=\Sigma_{p(j)}$,

(b) $\bigcup_{j=1}^{n} \Sigma_{j}=\bigcup_{j=1}^{n} \Sigma_{p(j)}$, and

(c) $\Sigma_{p(j)}=\Sigma_{p(k)}$ if and only if $p(j)=p(k)$.

The map $p$ is a choice of the indices of those, and only those, different $\Sigma_{j}$. Let $c_{1}:=\max \{p(j)\}_{j=1}^{n}$ and $c_{2}:=\max \left[\{p(j)\}_{j=1}^{n} \backslash\left\{c_{1}\right\}\right]$. We define the set

$$
\mathcal{F}_{P}:=\left\{\lambda \in \mathbb{C}: s_{c_{1}}(\lambda)=s_{c_{2}}(\lambda)\right\} \text {. }
$$

By virtue of Proposition 6, $\mathcal{F}_{P}$ has empty interior. Furthermore, if all eigenvalues of $P(\lambda)$ have geometric multiplicity equal to 1 , then

$$
\mathcal{F}_{P}=\left\{\lambda \in \mathbb{C}: s_{n}(\lambda)=s_{n-1}(\lambda)\right\} .
$$

Proposition 10. If all the eigenvalues of $P(\lambda)$ have geometric multiplicity equal to 1 , then either $\mathcal{F}_{P}=\emptyset$ or $\mathcal{F}_{P}$ lies on an algebraic curve (including the possibility of isolated points).

Proof. Let

$$
\hat{\mathcal{F}}=\left\{\lambda \in \mathbb{C}: s_{j}(\lambda)=s_{k}(\lambda), j \neq k\right\}
$$

so that $\mathcal{F}_{P} \subset \hat{\mathcal{F}}$. This set is the locus of all points $(x, y) \in \mathbb{R}^{2}$ such that the discriminant of the real polynomial in $S$ defined by (11) is zero. The hypothesis 
ensures that $\hat{\mathcal{F}} \neq \mathbb{C}$, and thus, either $\hat{\mathcal{F}}=\emptyset$ or $\hat{\mathcal{F}}$ is an algebraic curve. The result follows just because $\mathcal{F}_{P}$ is a subset of $\hat{\mathcal{F}}$.

In particular, $\mathcal{F}_{P}$ might include straight lines, single points, the empty set, or be a complicated set such as a Voronoi diagram (see Example 1 below).

Borrowing a geological term, we call the set $\mathcal{F}_{P}$ the set of fault points of $P(\lambda)$. In general, $\mathcal{F}_{P}$ will be made up of fault lines. The explicit determination of the fault lines of $P(\lambda)$ requires computations with determinants and discriminants, and is therefore unrealistic. However, the following considerations demonstrate the role that the fault lines frequently play in the study of pseudospectra.

Let $F_{\varepsilon}(x, y)$ be as in (12). As mentioned above, apart from $\lambda=0$, if $\nabla F(x, y)$ does not exist, then $x+i y \in \mathcal{F}_{P}$. At these points, the curve $\partial \Lambda_{\varepsilon}(P)$ will typically fail to have a tangent line. There are other points where the tangent line will be undefined, those where $\nabla F(x, y)=0$. In this case, there is a saddle point in the minimal singular value surface. These may or may not lie on $\mathcal{F}_{P}$ (see Section 6).

Example 3 below illustrates a case in which $\mathcal{F}_{P}$ is a singleton. In Example 4, $\mathcal{F}_{P}$ is empty but there is, nevertheless, a point at which $\partial \Lambda_{\varepsilon}(P)$ has no tangent. In Examples 1, 2, and $5, \mathcal{F}_{P}$ is, indeed, made up of fault lines.

Example 1. Let $A$ be an $n \times n$ normal matrix with eigenvalues $\left\{\lambda_{j}\right\}_{j=1}^{n}$. Then the fault lines of $P(\lambda)=I \lambda-A$ (i.e., for the standard eigenvalue problem) form the Voronoi diagram defined by $\left\{\lambda_{j}\right\}_{j=1}^{n}$ (i.e., the boundary of their Dirichlet tessellation).

Example 2. Naive experiments with diagonal matrix polynomials provide an insight on the possible structure of individual fault lines. For instance, let $P(\lambda)=$ $\operatorname{diag}\left\{\lambda^{2}-2 \lambda,(a-\lambda)(\lambda+2)\right\}$ and set $w(x)=1$. In Figure 1, we depict the evolution of the set $\mathcal{F}_{P}$ and $\partial \Lambda_{\varepsilon}(P)(\varepsilon=1 / \sqrt{2}, 1, \sqrt{3})$ for $a=-1, i, 1 / 2,1$. The fixed eigenvalues of $P(\lambda)$ are plotted as "+" and the perturbed eigenvalue $a$ is marked with a " $\diamond "$.

In general, an unbounded $\mathcal{F}_{P}$ appears to be more likely to occur. Nonetheless this set can also contain a compact smooth curve. In both of the lower figures, $\mathcal{F}_{P}$ consists of an unbounded curve, which is asymptotic to a vertical line, and a closed compact curve on the right half plane around the perturbed eigenvalue $a$. The curve $\partial \Lambda_{1}(P)$ has a self intersection at $\lambda=1$ for $a=-1, i, 1 / 2$. This can be shown from the fact that this part of the pseudospectrum depends only on the first diagonal entry of $P(\lambda)$. The self intersection disappears as soon as $a$ moves sufficiently close to 1 . There are critical values of $a$, where $1 \in \mathcal{F}_{P}$. Two of these critical values are $a=2 / 3$ and $a=4 / 3$.

Example 3. $\mathcal{F}_{P}$ can also be a singleton. In the left part of Figure 2, we depict $\partial \Lambda_{\varepsilon}(P)$ for the linear matrix polynomial

$$
P(\lambda)=\left[\begin{array}{ccc}
\lambda+3 i / 4 & 1 & 1 \\
0 & \lambda-5 / 4 & 1 \\
0 & 0 & \lambda+3 / 4
\end{array}\right],
$$

the weight function $w(x)=1$ and $\varepsilon^{2}=1 / 10,5 / 16,1 / 2$. The very special structure of this matrix polynomial ensures that $\mathcal{F}_{P}=\{0\}$. The boundary of the pseudospectrum does not have a tangent line at $\lambda=0$ when $\varepsilon=\sqrt{5 / 16}$. Compare with Example 4 below. 

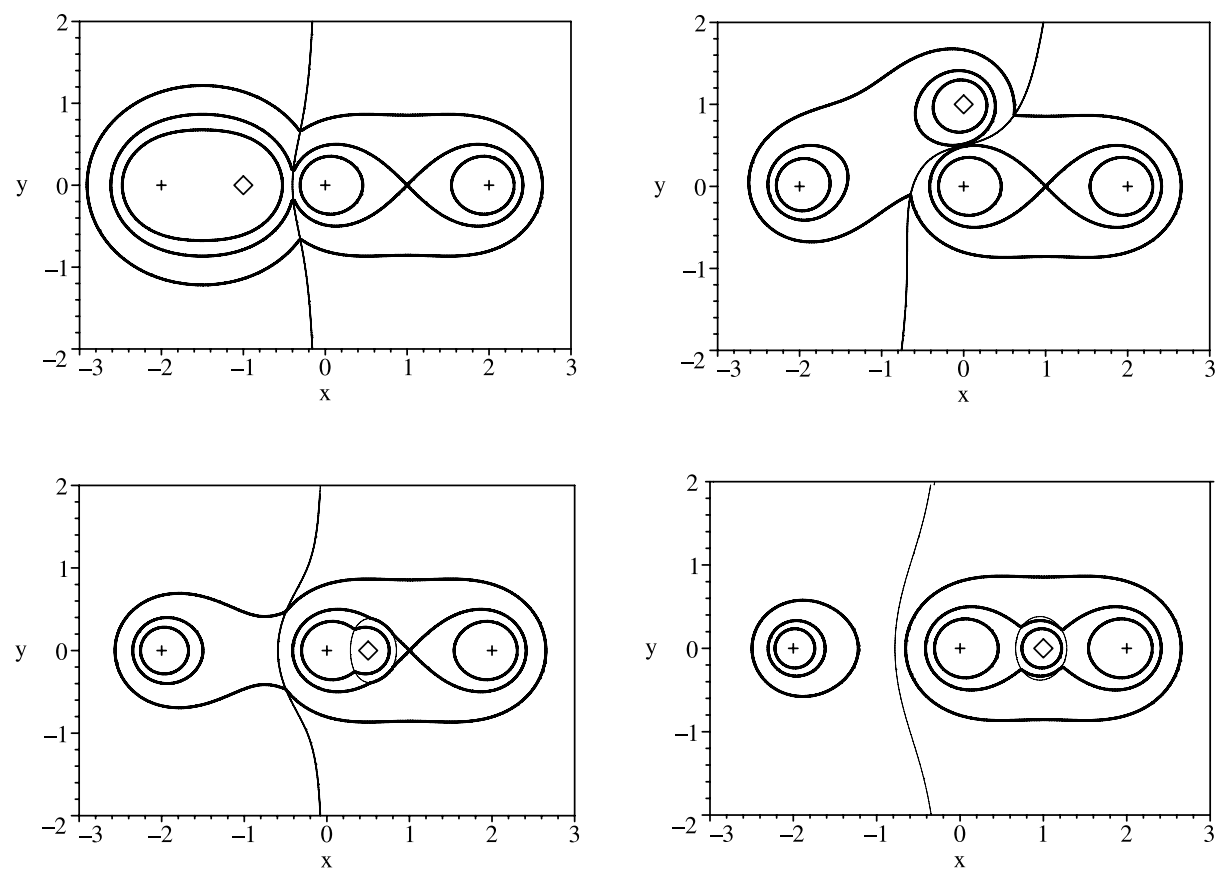

Figure 1 . The thin solid lines are $\mathcal{F}_{P}$. The thick solid lines are $\partial \Lambda_{\varepsilon}(P)$.
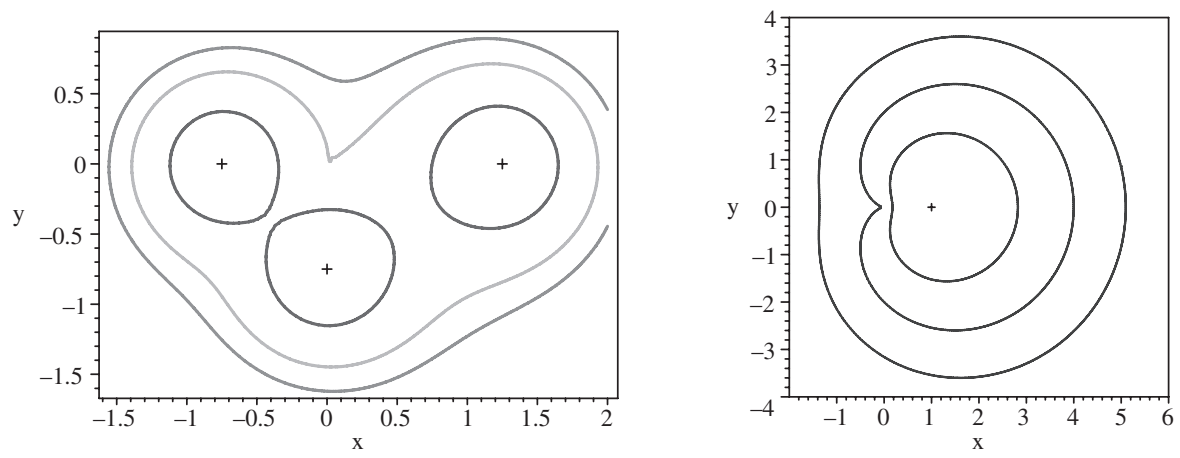

Figure 2. $\partial \Lambda_{\varepsilon}(P)$ has no tangent line at the origin for just one $\varepsilon$.

By construction, $\mathcal{F}_{P}$ is independent of $w(x)$. Therefore, the singularities occurring on $\partial \Lambda_{\varepsilon}(P)$ in places where the gradient of (12) fails to exist, are, with the possible exception of $\lambda=0$, independent of the chosen weights. In order to illustrate this remarkable fact, we consider two more examples.

Example 4. The set $\mathcal{F}_{P}$ might be empty but the smoothness of $\partial \Lambda_{\varepsilon}(P)$ might be broken at $\lambda=0$ due to the weight function. Indeed, let $n=1, P(\lambda)=(\lambda-1)^{2}$ and $w(x)=2 x+1$. Then $s_{1}(x+i y)=(x-1)^{2}+y^{2}$ and $\mathcal{F}_{P}=\emptyset$.

When $\varepsilon=1, F_{1}(x, y)=x^{2}+y^{2}-2 x-2 \sqrt{x^{2}+y^{2}}$. Hence, $F_{1}(x, y)=0$ if and only if

$$
(x-1)^{2}+y^{2} \geq 1 \text { and } y^{4}+2\left(x^{2}-2 x-2\right) y^{2}+\left(x^{4}-4 x^{3}\right)=0 .
$$



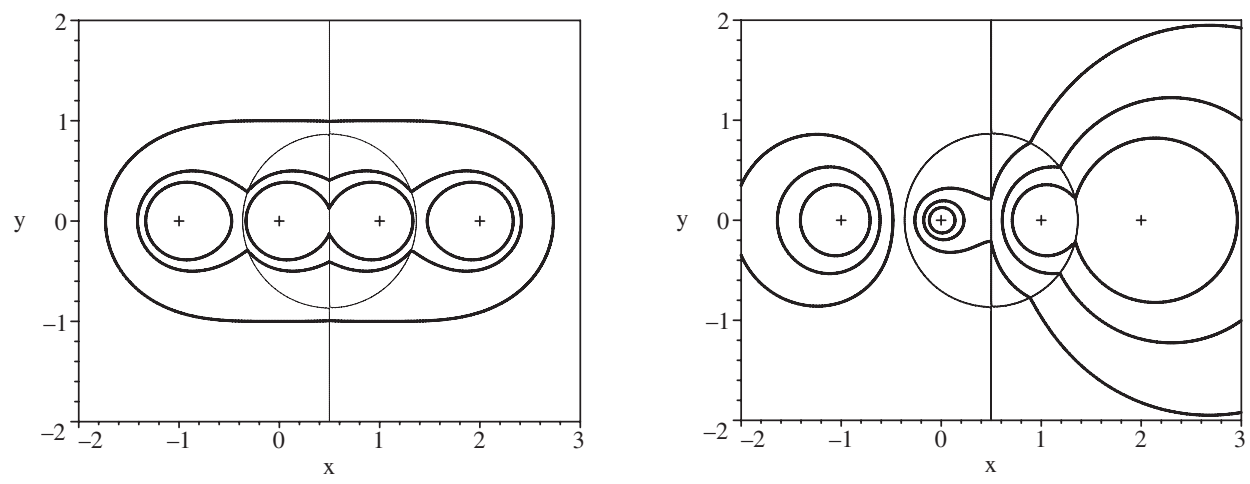

Figure 3 . The thin solid lines are $\mathcal{F}_{P}$. The thick solid lines are $\partial \Lambda_{\varepsilon}(P)$.

Thus, the curve $\partial \Lambda_{1}(P)$ has a parameterisation of the form

$$
y_{ \pm}(x)= \pm \sqrt{2+2 x-x^{2}-2 \sqrt{2 x+1}},-1 / 10 \leq x \leq 0,
$$

in a neighbourhood of the origin. As $\partial_{x} y_{+}(0)<0$ and $\partial_{x} y_{-}(0)>0,0 \in \partial \Lambda_{1}(P)$ is a singularity of Lipschitz type. The boundaries of $\Lambda_{\varepsilon}(P)$ for $\varepsilon=1 / 2,1,3 / 2$, are drawn in the right part of Figure 2 .

Example 5. Let $P(\lambda)=\operatorname{diag}\left\{\lambda^{2}-1, \lambda^{2}-2 \lambda\right\}$. In Figure 3, we depict $\mathcal{F}_{P}$ and $\partial \Lambda_{\varepsilon}(P)$ for $w(x)=1$ and $\varepsilon=\sqrt{3 / 5}, 1,2$ (left), and for $w(x)=x^{2}+x+1$ and $\varepsilon^{2}=1 / 20,1 / 10,1 / 5$ (right). Here, $\mathcal{F}_{P}$ comprises a circle centred at $(1 / 2,0)$ and the line $x=1 / 2$. As in the previous examples, "+" marks the locations of the eigenvalues of $P(\lambda)$.

All the above examples were designed in such a manner that both the fault points and the boundaries of pseudospectra can be constructed analytically either by hand or using algebraic computer packages. We produced Figures 1, 2 and 3 using commands provided in the standard distribution of Maple.

\section{ON THE NUMBER OF CONNECTED COMPONENTS}

Consider an $n \times n$ matrix polynomial $P(\lambda)$ as in (11), a real $\varepsilon>0$, and a weight function $w(\lambda)$ with $w(0)=w_{0}>0$. Theorem 2.3 of [12] will be useful in the remainder of the paper. First we examine the case in which $\sigma(P)$ contains multiple eigenvalues more carefully and without the restriction of boundedness. A technical lemma will assist in the argument.

Lemma 11. Suppose $A$ and $E$ are two $n \times n$ complex matrices such that the determinants $\operatorname{det} A$ and $\operatorname{det}(A+E)$ are nonzero. Then there is a continuous map $t \mapsto E(t) \in \mathbb{C}^{n \times n}, t \in[0,1]$, such that $E(0)=0, E(1)=E$, and

$$
\operatorname{det}(A+E(t)) \neq 0 \text { and }\|E(t)\| \leq\|E\|, \quad t \in[0,1] .
$$

Proof. Since $\operatorname{det} A \neq 0$ and $\operatorname{det}(A+E) \neq 0$, no eigenvalue of the pencil $A+t E$ can be equal to 0 or 1 (and some may be infinite). So it may be assumed that $\operatorname{det}(A+t E)$ has $s$ real zeros in the interval $(0,1)$, where $0 \leq s \leq n$.

If $s=0$, then the continuous map $t \mapsto t E, t \in[0,1]$, has the properties required by the lemma. If $s \geq 1$, then let $t_{1}<t_{2}<\cdots<t_{s}$ denote the zeros of 
$\operatorname{det}(A+t E)$ in $(0,1)$. For any $t_{j}(j=1,2, \ldots, s)$, the matrix $A+t_{j} E$ is singular, and for $\delta_{j}>0$ sufficiently small, we have

$$
\operatorname{det}\left[A+\left(t_{j}+\mathrm{e}^{i \theta} \delta_{j}\right) E\right] \neq 0 \quad \text { and } \quad\left\|\left(t_{j}+\mathrm{e}^{i \theta} \delta_{j}\right) E\right\| \leq\|E\|, \quad \theta \in[0,2 \pi] .
$$

In $[0,1]$, we replace each interval $\left[t_{j}-\delta_{j}, t_{j}+\delta_{j}\right]$ with the circular arc

$$
\mathcal{C}_{j}=\left\{t_{j}-\mathrm{e}^{i \theta} \delta_{j}: \theta \in[0, \pi]\right\},
$$

and consider the continuous curve

$\mathcal{S}=\left[0, t_{1}-\delta_{1}\right] \cup \mathcal{C}_{1} \cup\left[t_{1}+\delta_{1}, t_{2}-\delta_{2}\right] \cup \mathcal{C}_{2} \cup \cdots \cup\left[t_{s-1}+\delta_{s-1}, t_{s}-\delta_{s}\right] \cup \mathcal{C}_{s} \cup\left[t_{s}+\delta_{s}, 1\right]$

in the complex plane. For every continuous map $t \mapsto z(t) \in \mathcal{S}, t \in[0,1]$, such that $z(0)=0$ and $z(1)=1$, the map $t \mapsto z(t) E \in \mathbb{C}^{n \times n}, t \in[0,1]$, has the required properties.

We are now ready to establish our main result on the number of connected components of pseudospectra. We should remark that, when $\Lambda_{\varepsilon}(P)$ is bounded, the following theorem is a consequence of Theorem 2. Indeed, since $w(x)$ is a real polynomial, $w(|\lambda|)$ is a subharmonic function in $\mathbb{C}$ so, by Theorem $2, s(\lambda)^{-1} w(|\lambda|)$ is subharmonic in $\mathbb{C} \backslash \sigma(P)$. If $\Lambda_{\varepsilon}(P)$ had a connected component where there is no eigenvalue of $P(\lambda)$, then $s_{n}(\lambda) w(|\lambda|)^{-1}$ would have a local minimum in this component, which is impossible according to Theorem 2 .

Theorem 12. If the matrix polynomial $P(\lambda)$ has exactly $k(\leq n m)$ distinct eigenvalues (not necessarily simple), then for any $\varepsilon>0$, the pseudospectrum $\Lambda_{\varepsilon}(P)$ has at most $k$ connected components.

Proof. If $\Lambda_{\varepsilon}(P)=\mathbb{C}$, then there is nothing to prove. So assume that $\Lambda_{\varepsilon}(P) \neq \mathbb{C}$, and consider a perturbation

$$
Q(\lambda)=\left(P_{m}+\Delta_{m}\right) \lambda^{m}+\cdots+\left(P_{1}+\Delta_{1}\right) \lambda+P_{0}+\Delta_{0}
$$

in $\mathcal{B}(P, \varepsilon, w)$ with $\operatorname{det}\left(P_{m}+\Delta_{m}\right) \neq 0$. By Lemma 11, there is a continuous map $t \mapsto \Delta_{m}(t) \in \mathbb{C}^{n \times n}, t \in[0,1]$, such that $\Delta_{m}(0)=0, \Delta_{m}(1)=\Delta_{m}$, and

$$
\operatorname{det}\left(P_{m}+\Delta_{m}(t)\right) \neq 0 \text { and }\left\|\Delta_{m}(t)\right\| \leq\left\|\Delta_{m}\right\|, \quad t \in[0,1] .
$$

Hence, every member of the family

$$
Q_{t}(\lambda)=\left(P_{m}+\Delta_{m}(t)\right) \lambda^{m}+\cdots+\left(P_{1}+t \Delta_{1}\right) \lambda+P_{0}+t \Delta_{0}, \quad t \in[0,1],
$$

has exactly $n m$ eigenvalues, counting multiplicities. Moreover, all $Q_{t}(\lambda)(t \in[0,1])$ belong to $\mathcal{B}(P, \varepsilon, w)$. Their eigenvalues lie in $\Lambda_{\varepsilon}(P)$ and in trace continuous curves from the eigenvalues of $P(\lambda)\left(=Q_{0}(\lambda)\right)$ to the eigenvalues of $Q(\lambda)\left(=Q_{1}(\lambda)\right)$. Thus, as in the proof of Theorem 2.3 of [12, the set

$$
\Lambda_{0}=\left\{\mu \in \mathbb{C}: \operatorname{det} Q(\mu)=0, Q(\lambda) \in \mathcal{B}(P, \varepsilon, w), \operatorname{det}\left(P_{m}+\Delta_{m}\right) \neq 0\right\}
$$

has at most $k$ connected components determined by the $k$ distinct eigenvalues of $P(\lambda)$.

Now let $\lambda_{0}$ be an interior point of $\Lambda_{\varepsilon}(P)$, and let $R(\lambda)=\sum_{j=0}^{m} R_{j} \lambda^{j}$ be a perturbation in $\mathcal{B}(P, \varepsilon, w)$ with $\operatorname{det} R_{m}=0$, such that $\lambda_{0} \in \sigma(R)$. Since $\Lambda_{\varepsilon}(P) \neq$ $\mathbb{C}, R(\lambda)$ has less than $n m$ (finite) eigenvalues and, without loss of generality, we may assume that $R(\lambda) \in \partial \mathcal{B}\left(P, s_{n}\left(\lambda_{0}\right) w\left(\left|\lambda_{0}\right|\right)^{-1}, w\right) \subset \operatorname{Int}[\mathcal{B}(P, \varepsilon, w)]$ (see Lemma 8). Then $\lambda_{0}$ is also an eigenvalue of all matrix polynomials

$$
R_{\alpha}(\lambda)=\left(R_{m}+\alpha I\right) \lambda^{m}+R_{m-1} \lambda^{m-1}+\cdots+R_{1} \lambda+R_{0}-\left(\alpha \lambda_{0}^{m}\right) I, \quad \alpha \in \mathbb{C} \backslash\{0\},
$$


where $\operatorname{det}\left(R_{m}+\alpha I\right) \neq 0$ and $R_{\alpha}(\lambda) \in \mathcal{B}(P, \varepsilon, w)$ for sufficiently small $|\alpha|$, i.e., $\lambda_{0}$ lies in $\Lambda_{0}$. By Corollary $3, \Lambda_{\varepsilon}(P)$ does not have more connected components than $\Lambda_{0}\left(\subseteq \Lambda_{\varepsilon}(P)\right)$. Hence, $\Lambda_{\varepsilon}(P)$ has at most $k$ connected components.

In this theorem, recall that since the leading coefficient of $P(\lambda)$ is nonsingular, for $\varepsilon$ sufficiently small, $\Lambda_{\varepsilon}(P)$ has exactly $k$ bounded connected components. Thus, our upper bound for the number of connected components of $\Lambda_{\varepsilon}(P)$ is always attainable when $\Lambda_{\varepsilon}(P)$ is bounded.

Proposition 13. If $\Lambda_{\varepsilon}(P)$ is bounded, then any $Q(\lambda) \in \mathcal{B}(P, \varepsilon, w)$ has an eigenvalue in each of these components. Furthermore, $P(\lambda)$ and $Q(\lambda)$ have the same number of eigenvalues (counting algebraic multiplicities) in each connected component of $\Lambda_{\varepsilon}(P)$.

Proof. See Theorem 2.3 of [12].

\section{Multiple Eigenvalues of Perturbations}

In this section, we obtain necessary conditions for the existence of perturbations of $P(\lambda)$ with multiple eigenvalues. However, we first construct two perturbations of $P(\lambda)$ in $\mathcal{B}(P, \varepsilon, w)$, which are of special interest. They are used in an argument generalising that of Alam and Bora (Theorem 4.1 of [2]) for the standard eigenvalue problem.

Suppose that for a $\mu \in \Lambda_{\varepsilon}(P) \backslash \sigma(P)$, the (nonzero) minimum singular value of the matrix $P(\mu)$ has multiplicity $k \geq 1$. Also let

$$
s_{1}(\mu) \geq s_{2}(\mu) \geq \cdots \geq s_{n-k}(\mu)>s_{n-k+1}(\mu)=\cdots=s_{n}(\mu)>0
$$

be the singular values of $P(\mu)$ with associated left singular vectors $u_{1}, u_{2}, \ldots, u_{n}$ and associated right singular vectors $v_{1}, v_{2}, \ldots, v_{n}$. These singular vectors satisfy the relations $P(\mu) v_{j}=s_{j}(\mu) u_{j}$ for $j=1,2, \ldots, n$.

Define the $n \times n$ unitary matrix $\hat{Z}=\left[\begin{array}{llll}u_{1} & u_{2} & \cdots & u_{n}\end{array}\right]\left[\begin{array}{llll}v_{1} & v_{2} & \cdots & v_{n}\end{array}\right]^{*}$ and the

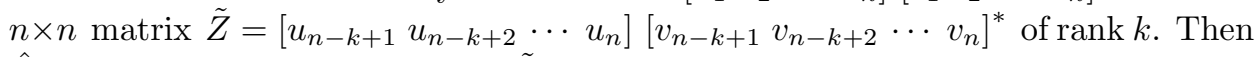
$\hat{Z} v_{j}=u_{j}$ for all $j=1,2, \ldots, n, \tilde{Z} v_{j}=u_{j}$ for all $j=n-k+1, n-k+2, \ldots, n$, and $\tilde{Z} v_{j}=0$ for all $j=1,2, \ldots, n-k$. Furthermore, the (nonsingular) matrix $\hat{E}=-s_{n}(\mu) \hat{Z}$ satisfies

$$
(P(\mu)+\hat{E}) v_{j}=s_{n}(\mu) u_{j}-s_{n}(\mu) u_{j}=0, \quad j=n-k+1, n-k+2, \ldots, n,
$$

and

$$
(P(\mu)+\hat{E}) v_{j}=s_{j}(\mu) u_{j}-s_{n}(\mu) u_{j} \neq 0, \quad j=1,2, \ldots, n-k .
$$

Similarly, the $(\operatorname{rank} k)$ matrix $\tilde{E}=-s_{n}(\mu) \tilde{Z}$ satisfies

$$
(P(\mu)+\tilde{E}) v_{j}=0, \quad j=n-k+1, n-k+2, \ldots, n,
$$

and

$$
(P(\mu)+\tilde{E}) v_{j}=s_{j}(\mu) u_{j} \neq 0, \quad j=1,2, \ldots, n-k .
$$

Note also that $\|\hat{E}\|=\|\tilde{E}\|=s_{n}(\mu)$.

Now define (for a given weight function $w(x)$ ) the matrices

$$
\hat{\Delta}_{j}=\left(\frac{\bar{\mu}}{|\mu|}\right)^{j} w_{j} w(|\mu|)^{-1} \hat{E}, \quad j=0,1, \ldots, m,
$$


and

$$
\tilde{\Delta}_{j}=\left(\frac{\bar{\mu}}{|\mu|}\right)^{j} w_{j} w(|\mu|)^{-1} \tilde{E}, \quad j=0,1, \ldots, m,
$$

where we set $\bar{\mu} /|\mu|=0$ when $\mu=0$. Then

$$
\sum_{j=0}^{m} \hat{\Delta}_{j} \mu^{j}=\left(\sum_{j=0}^{m} w_{j}|\mu|^{j}\right) w(|\mu|)^{-1} \hat{E}=\hat{E}
$$

and

$$
\sum_{j=0}^{m} \tilde{\Delta}_{j} \mu^{j}=\left(\sum_{j=0}^{m} w_{j}|\mu|^{j}\right) w(|\mu|)^{-1} \tilde{E}=\tilde{E} .
$$

Thus, for the (full rank) perturbation of $P(\lambda)$

$$
\hat{Q}(\lambda)=\left(P_{m}+\hat{\Delta}_{m}\right) \lambda^{m}+\cdots+\left(P_{1}+\hat{\Delta}_{1}\right) \lambda+P_{0}+\hat{\Delta}_{0}
$$

and the (lower rank) perturbation of $P(\lambda)$

$$
\tilde{Q}(\lambda)=\left(P_{m}+\tilde{\Delta}_{m}\right) \lambda^{m}+\cdots+\left(P_{1}+\tilde{\Delta}_{1}\right) \lambda+P_{0}+\tilde{\Delta}_{0},
$$

we have $\hat{Q}(\mu)=P(\mu)+\hat{E}$ and $\tilde{Q}(\mu)=P(\mu)+\tilde{E}$. From (14), (15), (16) and (17), it is clear that $\mu$ is an eigenvalue of the matrix polynomials $\hat{Q}(\lambda)$ and $\tilde{Q}(\lambda)$ with geometric multiplicity exactly $k$ and associated right eigenvectors $v_{n-k+1}, v_{n-k+2}, \ldots, v_{n}$.

Moreover, for every $j=0,1, \ldots, m$,

$$
\left\|\hat{\Delta}_{j}\right\|=w_{j} w(|\mu|)^{-1}\|\hat{E}\|=\frac{w_{j} s_{n}(\mu)}{w(|\mu|)} \leq \varepsilon w_{j}
$$

and

$$
\left\|\tilde{\Delta}_{j}\right\|=w_{j} w(|\mu|)^{-1}\|\tilde{E}\|=\frac{w_{j} s_{n}(\mu)}{w(|\mu|)} \leq \varepsilon w_{j} .
$$

Consequently, $\hat{Q}(\lambda)$ and $\tilde{Q}(\lambda)$ lie in $\mathcal{B}(P, \varepsilon, w)$, and the next result follows:

Proposition 14. Let $\mu \in \Lambda_{\varepsilon}(P) \backslash \sigma(P)$ and let the nonzero singular value $s_{n}(\mu)$ $(\leq \varepsilon w(|\mu|))$ of the matrix $P(\mu)$ have multiplicity $k \geq 1$. Then the perturbation $\hat{Q}(\lambda)$ in (18) and the perturbation $\tilde{Q}(\lambda)$ in (19) lie in $\mathcal{B}(P, \varepsilon, w)$ and have $\mu$ as an eigenvalue with geometric multiplicity equal to $k$.

Clearly, every fault point of $P(\lambda)$ in $\mathbb{C} \backslash \sigma(P)$ is a multiple eigenvalue of $\hat{Q}(\lambda)$ and $\tilde{Q}(\lambda)$ with geometric multiplicity greater than 1 . Furthermore, in the above discussion, note that for every $j=n-k+1, n-k+2, \ldots, n$,

$$
u_{j}^{*} P(\mu)=s_{n}(\mu) v_{j}^{*}
$$

and

$$
\begin{aligned}
u_{j}^{*}(P(\mu)+\hat{E}) & =u_{j}^{*} P(\mu)-s_{n}(\mu) u_{j}^{*} \hat{Z} \\
& =s_{n}(\mu) v_{j}^{*}-s_{n}(\mu)\left(\hat{Z}^{*} u_{j}\right)^{*} \\
& =s_{n}(\mu) v_{j}^{*}-s_{n}(\mu) v_{j}^{*}=0 .
\end{aligned}
$$

Similarly, for every $j=n-k+1, n-k+2, \ldots, n$, we have $u_{j}^{*}(P(\mu)+\tilde{E})=0$. Thus, $u_{n-k+1}, u_{n-k+2}, \ldots, u_{n}$ are left eigenvectors of the perturbations $\hat{Q}(\lambda)$ and $\tilde{Q}(\lambda)$ in (18) and (19), corresponding to $\mu$. 
The perturbations $\hat{Q}(\lambda)$ and $\tilde{Q}(\lambda)$ defined by (18) and (19) depend on $w(x)$ (which is considered fixed) and on the choice of $\mu$. It is also worth noting that for $\mu=0$ and a given weight function $w(x)$ with a constant coefficient $w_{0}>0$, the construction of $\hat{Q}(\lambda)$ and $\tilde{Q}(\lambda)$ is independent of the nonconstant part of $w(x)$ and requires only $w_{0}$. In the remainder of this paper, and without loss of generality, for the definition of $\hat{Q}(\lambda)$ and $\tilde{Q}(\lambda)$, we use the constant weight function $w_{c}(x)=w_{0}$ $(>0)$ instead of $w(x)$ whenever $\mu=0$.

Using Lemma 9, one can estimate the (spectral norm) distance from $P(\lambda)$ to the set of matrix polynomials that have a prescribed $\mu \notin \sigma(P)$ as an eigenvalue (cf. Lemma 3 of [16]).

Corollary 15. Suppose $\mu \notin \sigma(P)$, and let $\delta=s_{n}(\mu) w(|\mu|)^{-1}$. Then the perturbations $\hat{Q}(\lambda)$ and $\tilde{Q}(\lambda)$ lie on $\partial \mathcal{B}(P, \delta, w)$ and have $\mu$ as an eigenvalue. Moreover, for every $\varepsilon<\delta$, no perturbation of $P(\lambda)$ in $\mathcal{B}(P, \varepsilon, w)$ has $\mu$ as an eigenvalue.

Proposition 16. Let $\mu \in \sigma(P)$, and let $u, v \in \mathbb{C}^{n}$ be left and right eigenvectors of $P(\lambda)$ corresponding to $\mu$, respectively. If the derivative of $P(\lambda)$ satisfies $u^{*} P^{\prime}(\mu) v=$ 0 , then $\mu$ is a multiple eigenvalue of $P(\lambda)$.

Proof. If the geometric multiplicity of $\mu \in \sigma(P)$ is greater than 1 , then the proposition obviously holds. Hence, we assume that $\mu$ is an eigenvalue of $P(\lambda)$ with geometric multiplicity 1 . For every vector $y \in \mathbb{C}^{n}, u^{*} P(\mu) y=0$, and thus, $u \perp$ Range $[P(\mu)]$. Since $u \perp P^{\prime}(\mu) v$ and the dimension of Range $[P(\mu)]$ is $n-1$, it follows that the vector $P^{\prime}(\mu) v$ belongs to Range $[P(\mu)]$, i.e., there exists a $y_{\mu} \in \mathbb{C}^{n}$ such that

$$
P(\mu) y_{\mu}+P^{\prime}(\mu) v=0 .
$$

This shows that $\mu$ is a multiple eigenvalue of $P(\lambda)$ with the Jordan chain $\left\{v, y_{\mu}\right\}$ (see [9] for properties of Jordan chains of matrix polynomials). This implies that $\mu$ is a defective multiple eigenvalue of $P(\lambda)$.

Recall the function $F_{\varepsilon}(x, y) \equiv F_{\varepsilon}(x+i y)(x, y \in \mathbb{R})$ defined in (12).

Proposition 17. Suppose that for a point $\mu=x_{\mu}+i y_{\mu}$ of $\Lambda_{\varepsilon}(P) \backslash \sigma(P), s_{n}(\mu)$ is a simple singular value of $P(\mu)$, and $u_{\mu}, v_{\mu}$ are associated left and right singular vectors, respectively, assuming that $w(x)=w_{c}(x)\left(=w_{0}>0\right)$ when $\mu=0$. Let $\delta=s_{n}(\mu) w(|\mu|)^{-1}(\leq \varepsilon)$ and consider the perturbations $\hat{Q}(\lambda), \tilde{Q}(\lambda) \in \partial \mathcal{B}(P, \delta, w)$ defined by (18) and (19). If the gradient of the function $F_{\delta}(x, y) \equiv F_{\delta}(x+i y)$ at $\mu$ is zero, then $\mu$ is a defective eigenvalue of $\hat{Q}(\lambda)$ and $\tilde{Q}(\lambda)$ with geometric multiplicity 1.

Proof. Suppose $\mu \neq 0$, and let $\nabla F_{\delta}\left(x_{\mu}, y_{\mu}\right)=0$, or equivalently (see Lemma 4), let

$$
\operatorname{Re}\left(u_{\mu}^{*} \frac{\partial P(\mu)}{\partial x} v_{\mu}\right)=\delta \frac{\partial w(|\mu|)}{\partial x} \quad \text { and } \operatorname{Re}\left(u_{\mu}^{*} \frac{\partial P(\mu)}{\partial y} v_{\mu}\right)=\delta \frac{\partial w(|\mu|)}{\partial y}
$$

Since

we see that

$$
\frac{\partial P(\mu)}{\partial x}=P^{\prime}(\mu) \text { and } \frac{\partial P(\mu)}{\partial y}=i P^{\prime}(\mu)
$$

$$
\operatorname{Im}\left(u_{\mu}^{*} \frac{\partial P(\mu)}{\partial x} v_{\mu}\right)=-\operatorname{Re}\left(u_{\mu}^{*} \frac{\partial P(\mu)}{\partial y} v_{\mu}\right)
$$


Moreover,

$$
\frac{\partial w(|\mu|)}{\partial x}=\frac{x_{\mu}}{|\mu|} w^{\prime}(|\mu|) \text { and } \frac{\partial w(|\mu|)}{\partial y}=\frac{y_{\mu}}{|\mu|} w^{\prime}(|\mu|),
$$

and consequently,

$$
u_{\mu}^{*} P^{\prime}(\mu) v_{\mu}=u_{\mu}^{*} \frac{\partial P(\mu)}{\partial x} v_{\mu}=\delta \frac{\partial w(|\mu|)}{\partial x}-i \delta \frac{\partial w(|\mu|)}{\partial y}=\delta \frac{\bar{\mu}}{|\mu|} w^{\prime}(|\mu|) .
$$

Consider the perturbation

$$
\hat{Q}(\lambda)=\left(P_{m}+\hat{\Delta}_{m}\right) \lambda^{m}+\cdots+\left(P_{1}+\hat{\Delta}_{1}\right) \lambda+P_{0}+\hat{\Delta}_{0}
$$

in (18). Then $\hat{Q}(\lambda)$ lies on the boundary of the (compact) set $\mathcal{B}(P, \delta, w) \subseteq$ $\mathcal{B}(P, \varepsilon, w)$ and its derivative satisfies

$$
\begin{aligned}
u_{\mu}^{*} \hat{Q}^{\prime}(\mu) v_{\mu} & =u_{\mu}^{*} P^{\prime}(\mu) v_{\mu}+u_{\mu}^{*}\left(\sum_{j=1}^{m} j \hat{\Delta}_{j} \mu^{j-1}\right) v_{\mu} \\
& =\delta \frac{\bar{\mu}}{|\mu|} w^{\prime}(|\mu|)+\left(u_{\mu}^{*} \hat{E} v_{\mu}\right) \frac{w^{\prime}(|\mu|)}{w(|\mu|)} \frac{\bar{\mu}}{|\mu|} \\
& =\delta \frac{\bar{\mu}}{|\mu|} w^{\prime}(|\mu|)-\frac{s_{n}(\mu)}{w(|\mu|)} \frac{\bar{\mu}}{|\mu|} w^{\prime}(|\mu|) \\
& =\delta \frac{\bar{\mu}}{|\mu|} w^{\prime}(|\mu|)-\delta \frac{\bar{\mu}}{|\mu|} w^{\prime}(|\mu|)=0
\end{aligned}
$$

where $u_{\mu}$ and $v_{\mu}$ are left and right eigenvectors of $\hat{Q}(\lambda)$ corresponding to $\mu$, respectively (see Proposition 14 and the related discussion). The same is also true for the perturbation $\tilde{Q}(\lambda)$ in (19) and its derivative. By Propositions 14 and 16, $\mu$ is a multiple eigenvalue of $\hat{Q}(\lambda)$ and $\tilde{Q}(\lambda)$ with geometric multiplicity 1 .

For $\mu=0$, the proof is the same, keeping in mind that the constant weight function $w_{c}(x)=w_{0}(>0)$ is differentiable (with zero partial derivatives) at the origin.

\section{Multiple Points on $\partial \Lambda_{\varepsilon}(P)$ And Connected Components of $\Lambda_{\varepsilon}(P)$}

At first glance it may seem that multiple (crossing) points on $\partial \Lambda_{\varepsilon}(P)$ will be exceptional. However, when we consider the evolution of $\partial \Lambda_{\varepsilon}(P)$ as $\varepsilon$ increases, it is clear that, as disjoint components of $\Lambda_{\varepsilon}(P)$ expand, there will be critical values of $\varepsilon$ at which they meet and multiple points will be created.

Next, based on the results of the previous section, we show that multiple points of $\partial \Lambda_{\varepsilon}(P)$ are multiple eigenvalues of perturbations of $P(\lambda)$ on $\partial \mathcal{B}(P, \varepsilon, w)$ and, also, these perturbations can be constructed explicitly. (Recall that, when $\mu=0$, we use the constant weight function $w_{c}(x)=w_{0}>0$ for the definition of the perturbations $\hat{Q}(\lambda)$ and $\tilde{Q}(\lambda)$ in (18) and (19).)

Theorem 18. Suppose that, as the parameter $\varepsilon>0$ increases, two different connected components of $\Lambda_{\varepsilon}(P) \neq \mathbb{C}, \mathcal{G}_{1}$ and $\mathcal{G}_{2}$, meet at $\mu \in \mathbb{C}$. Then the following hold:

(i) If $\mu \neq 0$, then it is a multiple eigenvalue of the perturbations $\hat{Q}(\lambda), \tilde{Q}(\lambda) \in$ $\partial \mathcal{B}(P, \varepsilon, w)$ defined by (18) and (19).

(ii) If $\mu=0$ and $w(x)=w_{c}(x)\left(=w_{0}>0\right)$, then $\mu=0$ is a multiple eigenvalue of the perturbations $\hat{Q}(\lambda), \tilde{Q}(\lambda) \in \partial \mathcal{B}\left(P, \varepsilon, w_{c}\right)$. 
(iii) If $\mu=0, w(x) \neq w_{c}(x), \Lambda_{\varepsilon}(P)$ is bounded and the origin is the only intersection point of $\mathcal{G}_{1}$ and $\mathcal{G}_{2}$, then $\mu=0$ is a multiple eigenvalue of a perturbation on $\partial \mathcal{B}(P, \varepsilon, w)$.

Furthermore, in the first two cases, if $s_{n}(\mu)$ is a simple singular value of $P(\mu)$, then $\mu$ is a defective eigenvalue of $\hat{Q}(\lambda)$ and $\tilde{Q}(\lambda)$ with geometric multiplicity 1 .

Proof. Suppose that $s_{n}(\mu)(=\varepsilon w(|\mu|))$ is a multiple singular value of the matrix $P(\mu)$. Then by Proposition 14, the perturbations $\hat{Q}(\lambda), \tilde{Q}(\lambda) \in \partial \mathcal{B}(P, \varepsilon, w)$ have $\mu$ as a multiple eigenvalue of geometric multiplicity greater than 1 . Hence, we may assume that $s_{n}(\mu)$ is a simple singular value of $P(\mu)$, and consider the three cases of the theorem.

(i) Suppose $\mu \neq 0$, and recall (13). By virtue of Lemma 4 $F_{\varepsilon}(x, y)$ is real analytic in a neighbourhood of $\mu$. Furthermore, $\nabla F_{\varepsilon}(\mu)=0$, otherwise the implicit function theorem would ensure the existence of a smooth curve on a neighbourhood of $\mu$ parameterising $\partial \Lambda_{\varepsilon}(P)$ and contradict the fact that $\partial \mathcal{G}_{1} \cap \partial \mathcal{G}_{2}$ is a finite set (Theorem 17). Therefore, Proposition 17 yields the desired conclusion.

(ii) If $\mu=0$ and $w(x)=w_{c}(x)\left(=w_{0}>0\right)$, then the result follows by applying Proposition 17 as in case (i).

(iii) Suppose $\Lambda_{\varepsilon}(P)$ is bounded, $w(x) \neq w_{c}(x)$, and $\mu=0$ is the only intersection point of $\mathcal{G}_{1}$ and $\mathcal{G}_{2}$. By Proposition 13, for any positive $\delta<\varepsilon$, all the perturbations in $\mathcal{B}(P, \delta, w)$ have a constant number of eigenvalues in $\Lambda_{\delta}(P) \cap \mathcal{G}_{j}$, say $\kappa_{j}$, for $j=1,2$. Here and throughout this proof, eigenvalues are counted according to their algebraic multiplicities.

Define the sets

$$
\mathcal{B}=\{Q(\lambda) \in \mathcal{B}(P, \varepsilon, w): 0 \in \sigma(Q)\} \subseteq \partial \mathcal{B}(P, \varepsilon, w)
$$

and

$$
\mathcal{B}_{j}=\left\{Q(\lambda) \in \mathcal{B}: Q(\lambda) \text { has less than } \kappa_{j} \text { eigenvalues in } \mathcal{G}_{j} \backslash\{0\}\right\}, \quad j=1,2 .
$$

If $\mathcal{B}_{j}=\emptyset(j=1,2)$, then Proposition 13 and the continuity of the eigenvalues of matrix polynomials with respect to the entries of their coefficients imply that $0 \notin \mathcal{G}_{j}$; this is a contradiction. Hence, the sets $\mathcal{B}_{1}$ and $\mathcal{B}_{2}$ are both nonempty.

Now consider the constant weight function $w_{c}(\lambda)=w_{0}(>0)$ and the associated $\varepsilon$-pseudospectrum of $P(\lambda)$,

$$
\Lambda_{\varepsilon, w_{c}}(P)=\left\{\mu \in \mathbb{C}: \operatorname{det} Q(\mu)=0,\left\|\Delta_{0}\right\| \leq \varepsilon w_{0}, \Delta_{1}=\cdots=\Delta_{m}=0\right\} .
$$

Clearly, $\Lambda_{\varepsilon, w_{c}}(P) \subseteq \Lambda_{\varepsilon}(P)$ and $0 \in \partial \Lambda_{\varepsilon, w_{c}}(P)$. For any $j=1,2$, consider a perturbation

$$
Q_{j}(\lambda)=\left(P_{m}+\Delta_{m}\right) \lambda^{m}+\cdots+\left(P_{1}+\Delta_{1}\right) \lambda+P_{0}+\Delta_{0} \in \mathcal{B}_{j},
$$

and define the matrix polynomial

$$
Q_{j, c}(\lambda)=P_{m} \lambda^{m}+\cdots+P_{1} \lambda+P_{0}+\Delta_{0}=P(\lambda)+\Delta_{0} \in \mathcal{B} \cap \partial \mathcal{B}\left(P, \varepsilon, w_{c}\right)
$$

and the continuous trajectory

$$
Q_{j}(t ; \lambda)=\left(P_{m}+t \Delta_{m}\right) \lambda^{m}+\cdots+\left(P_{1}+t \Delta_{1}\right) \lambda+P_{0}+\Delta_{0} \in \mathcal{B}, \quad 0 \leq t \leq 1,
$$

with $Q_{j}(0 ; \lambda)=Q_{j, c}(\lambda)$ and $Q_{j}(1 ; \lambda)=Q_{j}(\lambda)$. If $\mu=0$ is a multiple eigenvalue of $Q_{j}(t ; \lambda)$ for some $t \in[0,1]$, then there is nothing to prove.

Let $\mu=0$ be a simple eigenvalue of $Q_{j}(t ; \lambda) \in \mathcal{B}$ for all $t \in[0,1]$. Since $\Lambda_{\varepsilon}(P)$ is bounded and the origin is the only intersection point of $\mathcal{G}_{1}$ and $\mathcal{G}_{2}$, by the continuity 
of the eigenvalues with respect to the coefficient matrices, it follows that all $Q_{j}(t ; \lambda)$ $(0 \leq t \leq 1)$ have exactly $\kappa_{j}-1$ eigenvalues in $\mathcal{G}_{j} \backslash\{0\}$, i.e., they lie in $\mathcal{B}_{j}$. Thus, $Q_{j, c}(\lambda) \in \mathcal{B}_{j}$. Moreover, again by Proposition 13 and the continuity of eigenvalues, an eigenvalue of the matrix polynomials $P(\lambda)+(1-t) \Delta_{0}(0 \leq t \leq 1)$ traces a continuous path in $\mathcal{G}_{j}$ connecting the origin with an eigenvalue of $P(\lambda)$. This means that the origin is an intersection point of $\Lambda_{\varepsilon, w_{c}}(P) \cap \mathcal{G}_{1}$ and $\Lambda_{\varepsilon, w_{c}}(P) \cap \mathcal{G}_{2}$. Hence, $\mu=0$ is a multiple point of $\partial \Lambda_{\varepsilon, c}(P)$, and as in (ii), it is a multiple eigenvalue of the perturbations $\hat{Q}(\lambda), \tilde{Q}(\lambda) \in \partial \mathcal{B}\left(P, \varepsilon, w_{c}\right) \subset \partial \mathcal{B}(P, \varepsilon, w)$.

Now we can generalise a theorem of Mosier concerning scalar polynomials (Theorem 3 of [14]).

Theorem 19. Suppose $\Lambda_{\varepsilon}(P)$ is bounded and $\mathcal{G}$ is a connected component of $\Lambda_{\varepsilon}(P)$. Then the matrix polynomial $P(\lambda)$ has more than one eigenvalue in $\mathcal{G}$ (counting multiplicities) if and only if there is a perturbation $Q(\lambda) \in \mathcal{B}(P, \varepsilon, w)$ with a multiple eigenvalue in $\mathcal{G}$.

Proof. For the converse part, it is clear that if a perturbation $Q(\lambda) \in \mathcal{B}(P, \varepsilon, w)$ has a multiple eigenvalue in $\mathcal{G}$, then by Proposition 13, $P(\lambda)$ has at least two eigenvalues in $\mathcal{G}$, counting multiplicities.

For the sufficiency, if the matrix polynomial $P(\lambda)$ has a multiple eigenvalue in $\mathcal{G}$, then there is nothing to prove. Thus, we assume that $P(\lambda)$ has two simple eigenvalues, $\lambda_{1}$ and $\lambda_{2}$, in $\mathcal{G}$. By the continuity of the eigenvalues with respect to the coefficient matrices, it follows that there is a positive $\delta \leq \varepsilon$, such that $\Lambda_{\delta}(P)$ has a (bounded) connected component $\mathcal{G}_{\delta} \subseteq \mathcal{G}$ that is composed of two compact sets, $\mathcal{G}_{1, \delta}$ and $\mathcal{G}_{2, \delta}$, with disjoint interiors and intersecting boundaries. Moreover, without loss of generality, we can assume that $\lambda_{1}$ and $\lambda_{2}$ lie in the interior of $\mathcal{G}_{1, \delta}$ and $\mathcal{G}_{2, \delta}$, respectively. Then the curve enclosing $\mathcal{G}_{\delta}$ either crosses itself or is tangent to itself at some point $\mu_{\delta} \in \mathbb{C} \backslash \sigma(P)$. The result follows from Theorem 18, Note that if $\mathcal{G}_{1, \delta}$ and $\mathcal{G}_{2, \delta}$ intersect at the origin and one other point, Theorem 18 does not apply at $\mu=0$, but it will at that other point.

\section{TWO NUMERICAL EXAMPLES}

We present two numerical examples, which illustrate the results of the previous section and suggest possible applications. The figures were drawn using the boundary-tracing algorithm described in [12].

Example 6. The spectrum of the $2 \times 2$ quadratic matrix polynomial

$$
P(\lambda)=\left[\begin{array}{cc}
(\lambda-1)^{2} & \lambda \\
0 & (\lambda-2)^{2}
\end{array}\right]=\left[\begin{array}{ll}
1 & 0 \\
0 & 1
\end{array}\right] \lambda^{2}+\left[\begin{array}{cc}
-2 & 1 \\
0 & -4
\end{array}\right] \lambda+\left[\begin{array}{ll}
1 & 0 \\
0 & 4
\end{array}\right]
$$

is $\sigma(P)=\{1,2\}$. Both eigenvalues are plotted as "+" in Figure 4, and have algebraic multiplicity equal to 2 and geometric multiplicity equal to 1 . The boundaries $\partial \Lambda_{\varepsilon}(P)$ for $w(x)=x^{2}+x+1$ (i.e., for perturbations measured in the absolute sense) and for $\varepsilon=0.005,0.0091,0.02,0.03$, are also sketched in Figure 4.

Assuming that the pseudospectrum $\Lambda_{0.0091}(P)$ is connected with one self-intersection $\mu=1.4145$ plotted as "o", this figure indicates that $\Lambda_{\varepsilon}(P)$ consists of two connected components for $\varepsilon<0.0091$, and that it is connected for $\varepsilon \geq 0.0091$. Moreover, the singular values of the matrix $P(\mu)$ are $s_{1}(\mu)=1.4650$ and $s_{2}(\mu)=$ 0.0402 , i.e., $s_{2}(\mu)$ is simple $(\mu$ is not a fault point of $P(\lambda)$ ) and the function

$$
F_{0.0091}(x, y) \equiv F_{0.0091}(x+i y)=s_{2}(x+i y)-0.0091 w(|x+i y|), \quad x, y \in \mathbb{R},
$$




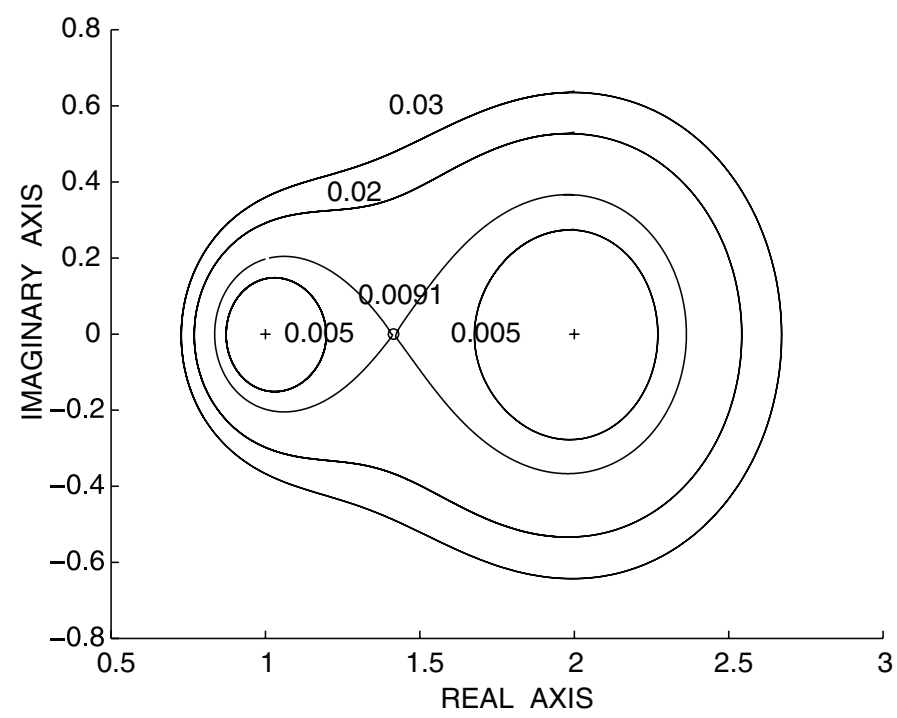

FiguRE 4. A single intersection point.

has zero gradient at the point $\mu$. Thus, by Proposition 17 and Theorem 18, two perturbations of $P(\lambda)$ on the boundary of $\mathcal{B}(P, 0.0091, w)$ that have $\mu$ as a defective eigenvalue are $\hat{Q}(\lambda)$ and $\tilde{Q}(\lambda)$ in (18) and (19), and can be easily constructed. Left and right singular vectors of $P(\mu)$ corresponding to $s_{1}(\mu)$ are

$$
u_{1}=\left[\begin{array}{l}
0.9726 \\
0.2325
\end{array}\right] \text { and } v_{1}=\left[\begin{array}{l}
0.1141 \\
0.9935
\end{array}\right],
$$

respectively, and left and right singular vectors of $P(\mu)$ corresponding to $s_{2}(\mu)$ are

$$
u_{2}=\left[\begin{array}{c}
-0.2325 \\
0.9726
\end{array}\right] \text { and } v_{2}=\left[\begin{array}{c}
-0.9935 \\
0.1141
\end{array}\right] \text {, }
$$

respectively.

The unitary matrix

$$
\hat{Z}=\left[\begin{array}{ll}
u_{1} & u_{2}
\end{array}\right]\left[\begin{array}{ll}
v_{1} & v_{2}
\end{array}\right]^{*}=\left[\begin{array}{cc}
0.3419 & 0.9397 \\
-0.9397 & 0.3419
\end{array}\right]
$$

satisfies

$$
\hat{Z} v_{1}=u_{1}, \quad u_{1}^{*} \hat{Z}=v_{1}^{*}, \quad \hat{Z} v_{2}=u_{2} \quad \text { and } \quad u_{2}^{*} \hat{Z}=v_{2}^{*}
$$

and the rank one matrix

$$
\tilde{Z}=u_{2} v_{2}^{*}=\left[\begin{array}{cc}
0.2310 & -0.0265 \\
-0.9663 & 0.1110
\end{array}\right]
$$

satisfies

$$
\tilde{Z} v_{1}=0, u_{1}^{*} \tilde{Z}=0, \quad \tilde{Z} v_{2}=u_{2} \text { and } u_{2}^{*} \tilde{Z}=v_{2}^{*} .
$$

We define the matrices

$$
\hat{\Delta}_{0}=\hat{\Delta}_{1}=\hat{\Delta}_{2}=\left(\mu^{2}+\mu+1\right)^{-1}\left(-s_{2}(\mu) \hat{Z}\right)=\left[\begin{array}{cc}
-0.0031 & -0.0086 \\
0.0086 & -0.0031
\end{array}\right]
$$


and the matrices

$$
\tilde{\Delta}_{0}=\tilde{\Delta}_{1}=\tilde{\Delta}_{2}=\left(\mu^{2}+\mu+1\right)^{-1}\left(-s_{2}(\mu) \tilde{Z}\right)=\left[\begin{array}{cc}
-0.0021 & 0.0002 \\
0.0088 & -0.0010
\end{array}\right],
$$

all with spectral norm 0.0091 . Then the perturbations

$$
\begin{gathered}
\hat{Q}(\lambda)=P(\lambda)+\left(\hat{\Delta}_{2} \lambda^{2}+\hat{\Delta}_{1} \lambda+\hat{\Delta}_{0}\right) \\
=\left[\begin{array}{cc}
0.9969 & -0.0086 \\
0.0086 & 0.9969
\end{array}\right] \lambda^{2}+\left[\begin{array}{cc}
-2.0031 & 0.9914 \\
0.0086 & -4.0031
\end{array}\right] \lambda+\left[\begin{array}{cc}
0.9969 & -0.0086 \\
0.0086 & 3.9969
\end{array}\right]
\end{gathered}
$$

and

$$
\begin{gathered}
\tilde{Q}(\lambda)=P(\lambda)+\left(\tilde{\Delta}_{2} \lambda^{2}+\tilde{\Delta}_{1} \lambda+\tilde{\Delta}_{0}\right) \\
=\left[\begin{array}{ll}
0.9979 & 0.0002 \\
0.0088 & 0.9990
\end{array}\right] \lambda^{2}+\left[\begin{array}{cc}
-2.0021 & 1.0002 \\
0.0088 & -4.0010
\end{array}\right] \lambda+\left[\begin{array}{cc}
0.9979 & 0.0002 \\
0.0088 & 3.9990
\end{array}\right]
\end{gathered}
$$

lie on $\partial \mathcal{B}(P, 0.0091, w)$ and have a multiple eigenvalue (approximately) equal to $\mu=1.4145$ with algebraic multiplicity 2 and geometric multiplicity 1 , confirming our results.

It is important to note that, by Theorems 18 and 19, pseudospectra yield a visual approximation of the distance to multiple eigenvalues, i.e., the spectral norm distance from an $n \times n$ matrix polynomial $P(\lambda)$ with a nonsingular leading coefficient and all its eigenvalues simple to $n \times n$ matrix polynomials with multiple eigenvalues. For a given weight function $w(x)$, this distance is defined by

$$
\begin{aligned}
\mathrm{r}(P) & :=\min \{\varepsilon>0: \exists Q(\lambda) \in \mathcal{B}(P, \varepsilon, w) \text { with multiple eigenvalues }\} \\
& \equiv \min \{\varepsilon>0: \exists Q(\lambda) \in \partial \mathcal{B}(P, \varepsilon, w) \text { with multiple eigenvalues }\}
\end{aligned}
$$

Then Theorems 18 and 19 imply the following result (see 2, 13, for the standard eigenvalue problem).

Corollary 20. Let $P(\lambda)$ be an $n \times n$ matrix polynomial as in (11) with a nonsingular leading coefficient and simple eigenvalues only.

(a) If $\Lambda_{\varepsilon}(P)$ is bounded, then

$$
\mathrm{r}(P)=\min \left\{\varepsilon>0: \Lambda_{\varepsilon}(P) \text { has less than } n \text { m connected components }\right\} \text {. }
$$

(b) If $\Lambda_{\varepsilon}(P)$ is unbounded and, as $\varepsilon$ increases from zero, its connected components meet at points different from the origin, then

$$
\mathrm{r}(P)=\min \left\{\varepsilon>0 \text { : the number of connected components of } \Lambda_{\varepsilon}(P) \text { decreases }\right\} \text {. }
$$

Example 7. Consider the $3 \times 3$ self-adjoint matrix polynomial

$$
P(\lambda)=\left[\begin{array}{lll}
1 & 0 & 0 \\
0 & 2 & 0 \\
0 & 0 & 5
\end{array}\right] \lambda^{2}+\left[\begin{array}{ccc}
0 & 0 & 0 \\
0 & 3 & -1 \\
0 & -1 & 6
\end{array}\right] \lambda+\left[\begin{array}{ccc}
2 & -1 & 0 \\
-1 & 3 & 0 \\
0 & 0 & 10
\end{array}\right]
$$

(see [12, Example 5.2]), which corresponds to a damped vibrating system. The boundaries of $\Lambda_{\varepsilon}(P)$ for $w(x)=\left\|A_{2}\right\| x^{2}+\left\|A_{1}\right\| x+\left\|A_{0}\right\|=5 x^{2}+6.3 x+10$ (i.e., for perturbations measured in a relative sense) and for $\varepsilon=0.02,0.05,0.1$, are drawn in Figure 5. The eigenvalues of $P(\lambda),-0.08 \pm i 1.45,-0.75 \pm i 0.86$ and $-0.51 \pm i 1.25$, are plotted as "+".

We learn from this figure and the above discussion that there exist an $\varepsilon_{1}=\mathrm{r}(P)$ in $(0.02,0.05)$ (for which, the pseudospectrum starts having less than six connected 


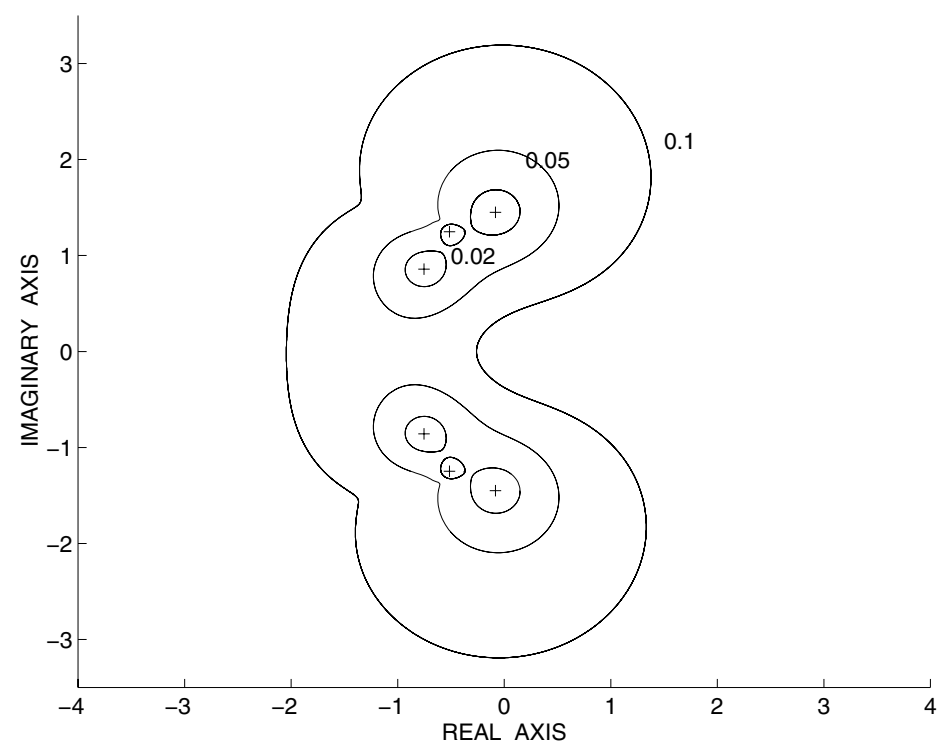

Figure 5. A damped vibrating system.

components) and an $\varepsilon_{2}$ in $(0.05,0.1)$ (for which, the pseudospectrum becomes connected) such that the following hold:

1. For every $\varepsilon<\varepsilon_{1}$, all the perturbations $Q(\lambda) \in \mathcal{B}(P, \varepsilon, w)$ have only simple eigenvalues.

2. For every $\varepsilon \in\left[\varepsilon_{1}, \varepsilon_{2}\right)$, some perturbations $Q(\lambda) \in \mathcal{B}(P, \varepsilon, w)$ have multiple nonreal eigenvalues (in a neighbourhood between the eigenvalues of $P(\lambda)$ in the open upper half-plane and in a neighbourhood between the eigenvalues of $P(\lambda)$ in the open lower half-plane), but no perturbation in $\mathcal{B}(P, \varepsilon, w)$ has multiple real eigenvalues.

3. For every $\varepsilon \geq \varepsilon_{2}$, some perturbations $Q(\lambda) \in \mathcal{B}(P, \varepsilon, w)$ have multiple real eigenvalues in the interval $[-2.1,-0.2]$.

\section{ACKNOWLEDGEMENTS}

The work of Peter Lancaster was supported in part by a grant from the Natural Sciences and Engineering Research Council of Canada. The work of Lyonell Boulton was supported in part by the Leverhulme Trust.

\section{REFERENCES}

[1] L.V. Ahlfors, Complex Analysis, McGraw Hill, New York (1953). MR510197(80c:30001)

[2] R. Alam and S. Bora, On sensitivity of eigenvalues and eigendecompositions of matrices, Linear Algebra Appl., 396 (2005) 273-301. MR2112210 (2005i:15013)

[3] L. Boulton, Non-variational approximation of discrete eigenvalues of self-adjoint operators, IMA J. Numer. Anal., 27 (2007), 102-121.

[4] S. Boyd and C.A. Desoer, Subharmonic functions and performance bounds on linear timeinvariant feedback systems, IMA J. Math. Control Inform., 2 (1985) 153-170. MR934961

[5] E. Brieskorn and H. Knörrer, Plane Algebraic Curves, Birkhäuser Verlag, Basel (1986). MR886476 (88a:14001) 
[6] E.B. Davies, Spectral enclosures and complex resonances for general self-adjoint operators, LMS J. Comput. Math., 1 (1998) 42-74. MR1635727(2000e:47043)

[7] J. Demmel, A counterexample for two conjectures about stability, IEEE Trans. Auto. Control, AC-32 (1987) 340-342.

[8] E. Gallestey, Computing spectral value sets using the subharmonicity of the norm of rational matrices, BIT, 38 (1998) 22-33. MR1621060 (99b:65045)

[9] I. Gohberg, L. Rodman, and P. Lancaster, Matrix Polynomials, Academic Press, Orlando, (1982). MR662418 (84c:15012)

[10] T. Kato, Perturbation Theory for Linear Operators, Springer-Verlag, New York (1980).

[11] K. Kendig, Elementary Algebraic Geometry, Springer Verlag, New York (1977). MR 0447222 (56:5537)

[12] P. Lancaster and P. Psarrakos, On the pseudospectra of matrix polynomials, SIAM J. Matrix Anal. Appl., 27 (2005) 115-129. MR2176811 (2006h:65058)

[13] A.N. Malyshev, A formula for the 2-norm distance from a matrix to the set of matrices with multiple eigenvalues, Numer. Math., 83 (1999) 443-454. MR.1715565 (2000i:65057)

[14] R.G. Mosier, Root neighbourhoods of a polynomial, Math. Comp., 47 (1986) 265-273. MR 842134 (87k:65056)

[15] J.-G. Sun, A note on simple non-zero singular values, J. Comput. Math., 6 (1988) 258-266. MR 967885(89i:15014)

[16] F. Tisseur, Backward error and condition of polynomial eigenvalue problems, Linear Algebra Appl., 309 (2000) 339-361. MR1758374 (2001c:65046)

[17] F. Tisseur and N.J. Higham, Structured pseudospectra for polynomial eigenvalue problems with applications, SIAM J. Matrix Anal. Appl., 23 (2001) 187-208. MR.1856605 (2002k:15024)

Department of Mathematics and the Maxwell Institute for Mathematical Sciences, Heriot-Watt University, Edinburgh EH14 2AS, United Kingdom

Department of Mathematics and Statistics, University of Calgary, Calgary AB, CANADA T2N $1 \mathrm{~N} 4$

Department of Mathematics, National Technical University, Zografou Campus, 5780 Athens, Greece 